\title{
The Equilibrium of Corrugated Plates and Shells
}

\author{
Samar Malek ${ }^{1}$ (D) Chris Williams ${ }^{2,3}$
}

Published online: 3 July 2017

(C) Kim Williams Books, Turin 2017

\begin{abstract}
Corrugations are used to stiffen sheet materials and in so doing they increase the bending and torsional stiffness in one direction and reduce the in plane membrane stiffness in the other. In this paper we examine the implications of this for the equilibrium of corrugated plate and shell structures. In particular we show that if the derivation is kept in terms of membrane strain energy then such a structure is statically determinate. As a result, a closed-form solution is obtained for a corrugated shell which is simple to solve numerically and could allow a designer to explore a range of different corrugation patterns and support conditions in the initial stage of design
\end{abstract}

Keywords Shells · Plates - Corrugations · Membrane action · Conceptual design

\section{Introduction}

In architecture, shell structures are used to span large areas like airports or stadiums because they require no intermediate support. Spanning such large areas is done most efficiently with shells because shell structures gain additional strength due to their curvature. However, shell structures are prone to buckling at a load below their

Samar Malek

malek@usna.edu

Chris Williams

C.J.K.Williams@bath.ac.uk; christopher.williams@chalmers.se

1 Department of Mechanical Engineering, United States Naval Academy, 590 Holloway Road, Annapolis, MD 21401, USA

2 Department of Architecture and Civil Engineering, University of Bath, Bath BA2 7AY, UK

3 Department of Architecture and Civil Engineering, Chalmers University of Technology, SE-412 96 Gothenburg, Sweden 
ideal theoretical capacity. Methods of preventing buckling include increasing the thickness of the shell or adding stiffening ribs. Another solution that is more architecturally and aesthetically interesting is to corrugate the shell's surface.

Corrugating shells is a natural solution as found in clamshells or ammonitida shells like those from Haeckel's Kunstformen der Natur (Art Forms in Nature) They have also been used in technology on the small and large scale. On the small scale, corrugation is used to stiffen and strengthen metal sheets which have been used on airplane and automotive fuselage. On a large scale, corrugations are used in shells like that of the Palazzetto dello Sport by Pier Luigi Nervi. Recent study has shown that corrugations can make an important contribution to the buckling resistance of shells (Malek 2012).

\section{Problem Statement}

The structural analysis of corrugated plates and shells is complex and relies on computational models and finite element analysis. For the early stages of design, simple analytic solutions are needed to help the engineer quickly assess the structural design. Furthermore, without close examination and understanding of the fundamental mechanics of corrugated plates and shells, erroneous results could be misinterpreted from a finite element analysis. Thus the aim of this paper is to derive and provide engineers with the equilibrium equations of corrugated plates and shells, ultimately proving that the structure is statically determinate and can be solved with closed-form solutions.

\section{Structural Mechanics Background}

Shell structures rely on two sorts of stiffness: membrane and bending stiffness (Calladine 1989). Membrane stiffness is the shell's resistance to changes in length of the surface. The word 'membrane' suggests a fabric-like material that can only work in tension, but in structural mechanics it is applied to all shells, working in tension and/or compression. For a solid shell of thickness $t$ made from a material of Young's modulus E, the membrane stiffness per unit width is equal to Et. This ignores Poisson's ratio effects where changes of length in one direction and membrane stress in the perpendicular direction interact.

The second kind of stiffness is bending stiffness, which is resistance to change of curvature. For the same solid shell the bending stiffness is $E t^{3} / 12$. If the size of a shell is represented by its span $S$, then the relative importance of bending to membrane stiffness is represented by the ratio $t^{2} / S^{2}$. It is much easier to bend a surface than it is to stretch it and therefore shells try to deform by reducing the amount of stretching to a minimum. Sometimes it is possible to deform with no stretching and this is called inextensional deformation. Lord Rayleigh showed that ringing bells deform primarily by inextensional deformation and hence the fundamental natural frequency is proportional to the square root of the bending stiffness (Strutt 1890). 
Thus shells rely on a combination of bending and membrane stiffness and this is particularly true for shells in compression that have to resist buckling (WoinowskyKrieger 1959; Wright 1965).

Corrugations are significant in structural terms because they increase the bending stiffness in the direction parallel to the corrugations, but decrease the membrane stiffness in the perpendicular direction due to what one might term the 'bellows' effect in which a change in length is accomplished by bending rather than stretching. Both the increase in bending stiffness and the decrease in membrane stiffness can be of many orders of magnitude, depending upon the ratio between the size of the corrugations and the thickness of the shell. Keith Seffen and Simon Guest and their colleagues in Cambridge (Korsunsky et al. 2009; Norman et al. 2008, 2009; Seffen 2012) have studied corrugated shells from the point of view of constructing a mechanism, however, we shall look at how a corrugated shell or plate can carry load.

The paper is mathematical, but the essential structural idea is relatively simple. A corrugated shell or plate is stiff in bending in the direction of the corrugations and flexible in bending in the perpendicular direction. First we will show that shells are statically determinate in membrane theory. Then we will derive equilibrium equations for a plate or shell with fine corrugations leading to a statically determinate structure.

\section{Membrane Action}

Figure 1 shows what we shall call a 'corrugated plate', that is a structure made from a thin sheet which is essentially flat, but upon which there are superimposed a wavelike system of corrugations. Figure $2 \mathrm{a}$ and $\mathrm{b}$ show two 'corrugated shells' that is again structures made from a thin sheet, but which are curved before superimposing wave-like corrugations. Figure $2 a$ has coarse corrugations whereas Fig. $2 b$ has fine corrugations. The mathematics in this paper will assume that the corrugations are 'extremely fine' so that in the limit we have a flat or curved surface whose local properties are determined by the corrugations. The idea is similar to the equivalent continuum used by Wright (1965).

We will assume that a corrugated shell or plate acts by membrane action, but with a limited amount of bending action to 'share the load across corrugations'. In

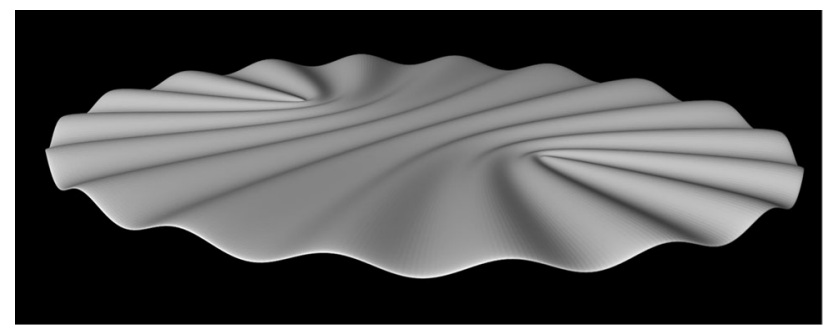

Fig. 1 Corrugated plate 

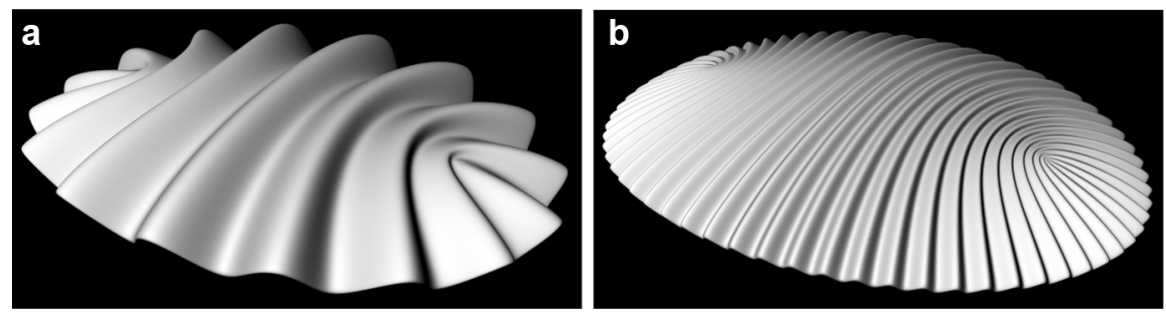

Fig. 2 a Shell with coarse corrugations. b Shell with fine corrugations

addition we shall assume that the load is vertical in which case we can use the Airy stress function in plan and only need to consider vertical equilibrium.

Equation (1) is equation $(f)$ from Article 113 in Chapter 14 of Timoshenko and Woinowsky-Krieger (1959), which gives references to the original derivation by Pucher.

$$
\frac{\partial^{2} F}{\partial x^{2}} \frac{\partial^{2} z}{\partial y^{2}}-2 \frac{\partial^{2} F}{\partial x \partial y} \frac{\partial^{2} z}{\partial x \partial y}+\frac{\partial^{2} F}{\partial y^{2}} \frac{\partial^{2} z}{\partial x^{2}}=q
$$

However, we shall use the symbol $\phi$ instead of $F$ to be consistent with Green and Zerna (1968). Thus we have:

$$
\frac{\partial^{2} \phi}{\partial x^{2}} \frac{\partial^{2} z}{\partial y^{2}}-2 \frac{\partial^{2} \phi}{\partial x \partial y} \frac{\partial^{2} z}{\partial x \partial y}+\frac{\partial^{2} \phi}{\partial y^{2}} \frac{\partial^{2} z}{\partial x^{2}}=q
$$

in which $\phi(x, y)$ is the Airy stress function, $z(x, y)$ is the height of the shell and $q(x, y)$ is the vertical load on the shell per unit plan area. The membrane stresses, expressed as the horizontal component of force per unit plan length are

$$
\begin{gathered}
\sigma_{x}=\frac{\partial^{2} \phi}{\partial y^{2}} \\
\sigma_{y}=\frac{\partial^{2} \phi}{\partial x^{2}} \\
\tau_{x y}=\tau_{y x}=-\frac{\partial^{2} \phi}{\partial x \partial y} .
\end{gathered}
$$

Equation (2) can be written in plane curvilinear coordinates as

$$
\left.\left.\varepsilon^{\alpha \gamma} \varepsilon^{\beta \rho} z\right|_{\alpha \beta} \phi\right|_{\gamma \rho}=q
$$

which is equation (11.2.11) of Green and Zerna (1968). Note that the curvilinear coordinates are referred to a plane $z=$ constant and therefore order of covariant differentiation is immaterial. Upon using the summation convention we have

$$
\varepsilon^{12} \varepsilon^{12}\left(\left.\left.z\right|_{11} \phi\right|_{22}-\left.\left.2 z\right|_{12} \phi\right|_{12}+\left.\left.z\right|_{22} \phi\right|_{11}\right)=q
$$

which is the same as Eq. (2), if we swap $\phi$ and $z$. 
Let us define the shape of a corrugated shell by

$$
z=Z+h \cos \psi
$$

in which $Z$ gives the overall shape and $h \cos \psi$ gives the corrugations which are assumed to be sinusoidal. $Z, h$ and $\psi$ are functions of the curvilinear coordinates $\theta^{1}$ and $\theta^{2}$ and therefore of $x$ and $y$. In the case of a corrugated plate we would have $Z=0$.

We can form the second covariant derivative using the same rules as for the partial derivative to give

$$
\left.z\right|_{\alpha \beta}=\left.Z\right|_{\alpha \beta}+\left(\left.h\right|_{\alpha \beta}-\left.\left.h \psi\right|_{\alpha} \psi\right|_{\beta}\right) \cos \psi-\left(\left.\left.h\right|_{\alpha} \psi\right|_{\beta}+\left.\left.\psi\right|_{\alpha} h\right|_{\beta}+\left.h \psi\right|_{\alpha \beta}\right) \sin \psi .
$$

Now let us assume that the stress function balancing a vertical load is of the form

$$
\phi=\Phi+f \cos \psi+F \sin \psi
$$

leading to

$$
\begin{gathered}
\left.\phi\right|_{\gamma \rho}=\left.\Phi\right|_{\gamma \rho}+\left(\left.f\right|_{\gamma \rho}-\left.\left.f \psi\right|_{\gamma} \psi\right|_{\rho}+\left.\left.F\right|_{\gamma} \psi\right|_{\rho}+\left.\left.\psi\right|_{\gamma} F\right|_{\rho}+\left.F \psi\right|_{\gamma \rho}\right) \cos \psi \\
+\left(\left.F\right|_{\gamma \rho}-\left.\left.F \psi\right|_{\gamma} \psi\right|_{\rho}-\left.\left.f\right|_{\gamma} \psi\right|_{\rho}-\left.\left.\psi\right|_{\gamma} f\right|_{\rho}-\left.f \psi\right|_{\gamma \rho}\right) \sin \psi
\end{gathered}
$$

Upon substituting Eqs. (9) and (11) into Eq. (6) we have

$$
q=A+B \cos \psi+C \sin \psi+D \cos ^{2} \psi+E \cos \psi \sin \psi+G \sin ^{2} \psi
$$

and we shall assume that the local bending stiffness of the shell or plate can 'share the load across corrugations' to give the 'local average load'

$$
\bar{q}=A+\frac{D}{2}+\frac{G}{2} .
$$

This is based upon the fact that the average value of $\cos \psi, \sin \psi$ and $\cos \psi \sin \psi$ is zero, whereas the average value of $\cos ^{2} \psi$ and $\sin ^{2} \psi$ is $\frac{1}{2}$.

If we assume that the unknowns are $\Phi, f$ and $F$ in Eq. (10) then we need two more equations in addition to Eq. (14). It would seem reasonable to stipulate that the coefficients of $\cos \psi$ and $\sin \psi$ are both zero in Eq. (13) so that

$$
\begin{aligned}
& B=0 \\
& C=0 .
\end{aligned}
$$

Substituting Eq. (15) into Eq. (13) using Eqs. (6), (9) and (11) gives

$$
\varepsilon^{\alpha \gamma} \varepsilon^{\beta \rho}\left(\begin{array}{c}
\left.Z\right|_{\alpha \beta}\left(\left.f\right|_{\gamma \rho}-\left.\left.f \psi\right|_{\gamma} \psi\right|_{\rho}+\left.\left.F\right|_{\gamma} \psi\right|_{\rho}+\left.\left.\psi\right|_{\gamma} F\right|_{\rho}+\left.F \psi\right|_{\gamma \rho}\right) \\
+\left.\left(\left.h\right|_{\alpha \beta}-\left.\left.h \psi\right|_{\alpha} \psi\right|_{\beta}\right) \Phi\right|_{\gamma \rho}
\end{array}\right)=0
$$

and 


$$
\varepsilon^{\alpha \gamma} \varepsilon^{\beta \rho}\left(\begin{array}{l}
\left.Z\right|_{\alpha \beta}\left(\left.F\right|_{\gamma \rho}-\left.\left.F \psi\right|_{\gamma} \psi\right|_{\rho}-\left.\left.f\right|_{\gamma} \psi\right|_{\rho}-\left.\left.\psi\right|_{\gamma} f\right|_{\rho}-\left.f \psi\right|_{\gamma \rho}\right) \\
+\left.\left(\left.\left.h\right|_{\alpha} \psi\right|_{\beta}+\left.\left.\psi\right|_{\alpha} h\right|_{\beta}+\left.h \psi\right|_{\alpha \beta}\right) \Phi\right|_{\gamma \rho}
\end{array}\right)=0 .
$$

Finally using Eqs. (6), (9) and (11), Eq. (14) becomes

$$
\begin{gathered}
\bar{q}=\left.\left.\varepsilon^{\alpha \gamma} \varepsilon^{\beta \rho} Z\right|_{\alpha \beta} \Phi\right|_{\gamma \rho} \\
+\frac{1}{2} \varepsilon^{\alpha \gamma} \varepsilon^{\beta \rho}\left(\left.h\right|_{\alpha \beta}-\left.\left.h \psi\right|_{\alpha} \psi\right|_{\beta}\right)\left(\begin{array}{c}
\left.f\right|_{\gamma \rho}-\left.\left.f \psi\right|_{\gamma} \psi\right|_{\rho} \\
+\left.\left.F\right|_{\gamma} \psi\right|_{\rho}+\left.\left.\psi\right|_{\gamma} F\right|_{\rho}+\left.F \psi\right|_{\gamma \rho}
\end{array}\right) \\
-\frac{1}{2} \varepsilon^{\alpha \gamma} \varepsilon^{\beta \rho}\left(\left.\left.h\right|_{\alpha} \psi\right|_{\beta}+\left.\left.\psi\right|_{\alpha} h\right|_{\beta}+\left.h \psi\right|_{\alpha \beta}\right)\left(\begin{array}{c}
\left.F\right|_{\gamma \rho}-\left.\left.F \psi\right|_{\gamma} \psi\right|_{\rho} \\
-\left.\left.f\right|_{\gamma} \psi\right|_{\rho}-\left.\left.\psi\right|_{\gamma} f\right|_{\rho}-\left.f \psi\right|_{\gamma \rho}
\end{array}\right) .
\end{gathered}
$$

\section{Approximation}

In order to make Eqs. (17), (18) and (19) tractable, let us assume that we have fine, short wavelength corrugations so that $\psi$ varies quickly over the surface. We will therefore only take the highest order term in $\psi$ Eqs. (17), (18) and (19) to give

$$
\begin{gathered}
\left.\left.\varepsilon^{\alpha \gamma} \varepsilon^{\beta \rho} \psi\right|_{\alpha} \psi\right|_{\beta}\left(\left.Z\right|_{\gamma \rho} f+\left.h \Phi\right|_{\gamma \rho}\right)=0, \\
\left.\left.\left.\varepsilon^{\alpha \gamma} \varepsilon^{\beta \rho} \psi\right|_{\alpha} \psi\right|_{\beta} Z\right|_{\gamma \rho} F=0
\end{gathered}
$$

and

$$
\bar{q}=\left.\left.\varepsilon^{\alpha \gamma} \varepsilon^{\beta \rho} Z\right|_{\alpha \beta} \Phi\right|_{\gamma \rho}-\left.\left.\left.\frac{1}{2} \varepsilon^{\alpha \gamma} \varepsilon^{\beta \rho} \psi\right|_{\alpha} \psi\right|_{\beta}(h f)\right|_{\gamma \rho}
$$

We are not interested in Eq. (23) since it only gives us $F$ (unless $\left.\left.\left.\varepsilon^{\alpha \gamma} \varepsilon^{\beta \rho} \psi\right|_{\alpha} \psi\right|_{\beta} Z\right|_{\gamma \rho}=0$ ) which does not appear in Eqs. (22) and (24). Equations (22) and (24) are two simultaneous differential equations in the unknowns $\Phi$ and $f$ and therefore the corrugated shell structure is statically determinate.

A simple example is the corrugated arch shown on Fig. 3. Clearly the arch carries a uniform load by a uniform axial stress, but asymmetric bending is taken by the corrugations giving bending stiffness.

\section{Application to Plates}

In the case of a corrugated plate Eq. (24) reduces to

$$
\bar{q}=-\left.\left.\left.\frac{1}{2} \varepsilon^{\alpha \gamma} \varepsilon^{\beta \rho} \psi\right|_{\alpha} \psi\right|_{\beta}(h f)\right|_{\gamma \rho}
$$

or 


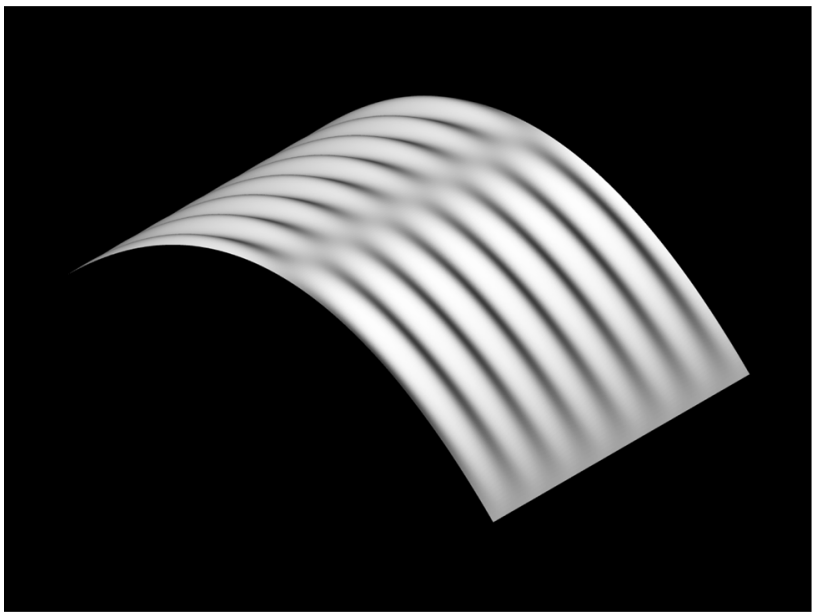

Fig. 3 Corrugated arch

$$
q=-\left(\frac{\partial \psi}{\partial x}\right)^{2} \frac{\partial^{2}(h f)}{\partial y^{2}}+2 \frac{\partial \psi}{\partial x} \frac{\partial \psi}{\partial y} \frac{\partial^{2}(h f)}{\partial x \partial y}-\left(\frac{\partial \psi}{\partial y}\right)^{2} \frac{\partial^{2}(h f)}{\partial x^{2}}
$$

in Cartesian coordinates. This is a parabolic differential equation in $f$, although actually only the product $h f$ occurs in the equation, so that we can treat $h f$ as the unknown and only specify the plan shape of the corrugations via $\psi$.

\section{Straight Corrugations}

The case of straight corrugations is especially simple and if we substitute

$$
\begin{aligned}
& z=h(x, y) \cos \left(\frac{2 \pi x}{\lambda}\right) \\
& \phi=f(x, y) \cos \left(\frac{2 \pi x}{\lambda}\right)
\end{aligned}
$$

into Eq. (26) we obtain

$$
\bar{q}=-\frac{1}{2}\left(\frac{2 \pi}{\lambda}\right)^{2} \frac{\partial^{2}(h f)}{\partial y^{2}}
$$

which has the solution

$$
h f=\bar{q}\left(\frac{\lambda}{2 \pi}\right)^{2}\left(y^{2}-\frac{L^{2}}{4}\right)
$$

where $L$ is the span.

Figure 4a shows $z$ below and $\phi$ above for a corrugated sheet. We could of course swap $z$ below and $\phi$, or if we prefer we could use the same shape for both as in 

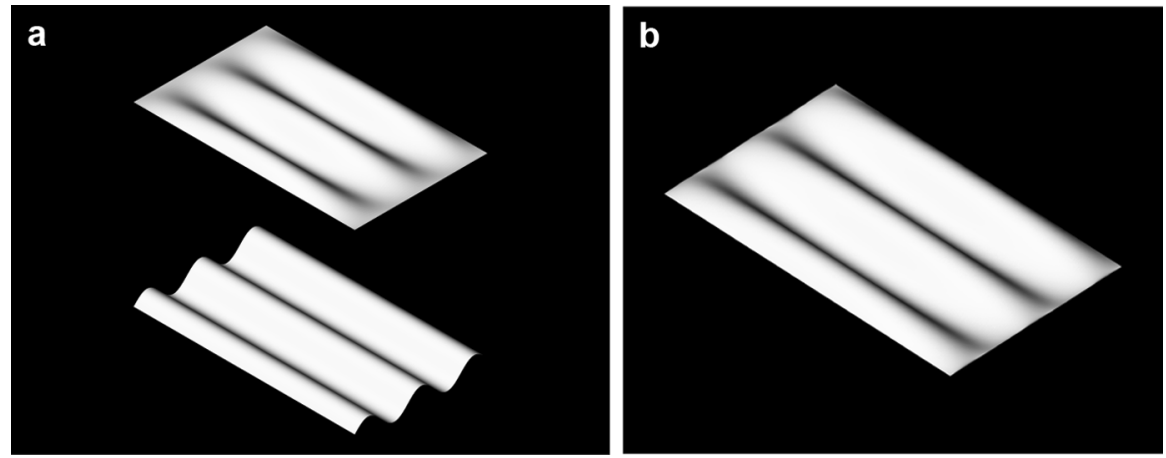

Fig. 4 a Corrugated plate $z$ below and $\phi$ above or vice versa. b Same function for $z$ and $\phi$

Fig. 4b. The stress given by the stress function used in Fig. 4a can be compared to results using the finite element method described in Molina et al. (2014).

\section{Conclusions and Further Work}

We have demonstrated that corrugated plates and shells can be considered to be essentially statically determinate. If they are not sufficiently supported, then they will be mechanisms and this could be a further line of research, which could be compared with the results from Cambridge (Korsunsky et al. 2009; Norman et al. 2008, 2009; Seffen 2012).

The differential equations (25) and (26) are simple to solve numerically and a range of different corrugation patterns and support conditions could be investigated. Corrugation patterns would be chosen partly on aesthetics and partly on functional grounds. However, this research will allow engineers and designers to quantify the effects of corrugation on the structural performance, making more informed decisions during the early phase of design.

\section{References}

Calladine, C. 1989. Theory of Shell Structures. Cambridge: Cambridge University Press.

Green, A. E. and Zerna, W. 1968. Theoretical Elasticity. Oxford: Oxford University Press, 2nd edition. Korsunsky, A. M., Dini, D., Sih, G. C., Gentilinia, C., Nobilea, L., and Seffen, K. A. 2009. Mesomechanics 2009 numerical analysis of morphing corrugated plates. Procedia Engineering, 1(1):79-82.

Malek, S. R. 2012. The effect of geometry and topology on the mechanics of grid shells. PhD thesis, Massachusetts Institute of Technology.

Molina, J. C., Fiorelli, J., and Savastano Jr., H. 2014. Analysis of the stresses in corrugated sheets under bending. Materials Research, 17:338 - 345.

Norman, A., Seffen, K., and Guest, S. 2008. Multistable corrugated shells. Proceedings of the Royal Society of London A: Mathematical, Physical and Engineering Sciences, 464(2095):1653-1672.

Norman, A., Seffen, K., and Guest, S. 2009. Morphing of curved corrugated shells. International Journal of Solids and Structures, 46(7-8):1624-1633. 
Seffen, K. A. 2012. Compliant shell mechanisms. Philosophical Transactions of the Royal Society of London A: Mathematical, Physical and Engineering Sciences, 370(1965):2010-2026.

Strutt, J. 1890. On bells. Philosophical Magazine, 29:1-17.

Timoshenko, S. P., and Woinowsky-Krieger, S. 1959. Theory of plates and shells. New York: McGrawHill.

Wright, D. T. 1965. Membrane forces and buckling in reticulated shells. American Society of Civil Engineers Proceedings Journal of the Structural Division, 91(10):173-201.

Samar Malek United States Naval Academy. Samar is a structural engineer with an expertise in structural and computational mechanics, and gridshells. She completed her Ph.D. and S.M. in Structures and Materials at MIT where she was also a lecturer in the Department of Architecture. Dr. Malek is a Marshall-Sherfield Scholar and completed her post-doctorate at the University of Bath Department of Architecture and Civil Engineering. She has practiced as a structural engineer at Thornton Tomasetti, NYC and consulted on gridshell projects for Atelier One, London. Her research interests include computational methods in conceptual structural design, gridshells, deployable shelters and biomimcry in architecture and engineering.

Chris Williams Chalmers University and Bath University. Chris worked with Ted Happold and Ian Liddell at Ove Arup and Partners on the Frei Otto gridshells in Mannheim. This lead to his research interest in the relationship between geometry and structural action, and collaboration on a number of projects including the British Museum Great Court Roof (Buro Happold and Foster + Partners), the Savill Building (Buro Happold and Glenn Howells Architects), Gardens by the Bay glasshouses (Atel- ier One, Wilkinson Eyre and Grant Associates) and the Netherlands Maritime Museum (Ney + Partners). 